\title{
NOTE ON CONVERGENCE TESTS FOR SERIES AND ON STIELTJES INTEGRATION BY PARTS.
}

\author{
BY PROFESSOR R. D. CARMICHAEL.
}

(Read before the American Mathematical Society September 4, 1919.)

THE obvious identity

$$
\begin{gathered}
{\left[c_{k}^{(1)} c_{k}^{(2)} \cdots c_{k}{ }^{(n)}-a_{k}{ }^{(1)} a_{k}{ }^{(2)} \cdots a_{k}{ }^{(n)}\right]=\left[a_{k}{ }^{(2)} a_{k}{ }^{(3)} \cdots\right.} \\
\left.a_{k}{ }^{(n)}\left(c_{k}{ }^{(1)}-a_{k}{ }^{(1)}\right)\right]+\sum_{i=2}^{n-1}\left[c_{k}^{(1)} \cdots c_{k}^{(i-1)} a_{k}{ }^{(i+1)} \cdots a_{k}^{(n)}\right. \\
\left.\left(c_{k}^{(i)}-a_{k}^{(i)}\right)\right]+\left[c_{k}^{(1)} \cdots c_{k}^{(n-1)}\left(c_{k}^{(n)}-a_{k}^{(n)}\right)\right]
\end{gathered}
$$

may serve in several ways for the investigation of the convergence of series. In each it is to be used as a means of relating the $n+1$ sums of the first $m$ terms of $n+1$ different series the general $k$ th terms of which in order are the $n+1$ bracketed expressions in the foregoing identity. It is obvious that the convergence of any $n$ of these series implies the convergence of the remaining one. Moreover, when they all converge, the identity leads to an obvious relation among the $n+1$ sums.

The effectiveness of the whole class of theorems arising thus lies in the fact that the series whose convergence is asserted may be, and in important cases is, conditionally convergent while the auxiliary series are absolutely convergent.

To arrive at a special instance of the theorems thus indicated, let us put $a_{k}{ }^{(i)}=c_{k-1}{ }^{(i)}$ with $c_{0}{ }^{(i)}=0$ and sum in (1) with respect to $k$ from 1 to $m$. Then we have

$$
\begin{aligned}
c_{m}{ }^{(1)} c_{m}{ }^{(2)} & \cdots c_{m}{ }^{(n)}=\sum_{k=1}^{m} c_{k-1}{ }^{(2)} c_{k-1}{ }^{(3)} \cdots c_{k-1}{ }^{(n)}\left(c_{k}{ }^{(1)}-c_{k-1}{ }^{(1)}\right) \\
& +\sum_{i=2}^{n-1} \sum_{k=1}^{m} c_{k}{ }^{(1)} \cdots c_{k}{ }^{(i-1)} c_{k-1}{ }^{(i+1)} \cdots c_{k-1}{ }^{(n)} \\
& \times\left(c_{k}{ }^{(i)}-c_{k-1}{ }^{(i)}\right)+\sum_{k=1}^{m} c_{k}{ }^{(1)} \cdots c_{k}{ }^{(n-1)}\left(c_{k}{ }^{(n)}-c_{k-1}{ }^{(n)}\right) .
\end{aligned}
$$

From this relation it is easy to see that we have the following result:*

* The theorems of the paper are given in forms suitable for ready use where applicable. In view of the general remark in the first paragraph it is clear that stronger (though less simply stated) theorems may be obtained from identity (2). A similar remark may be made about other results in the paper. 
Theorem I. The convergence of the series

$$
\sum_{k=1}^{\infty} c_{k-1}{ }^{(2)} c_{k-1}{ }^{(3)} \cdots c_{k-1}^{(n)}\left(c_{k}^{(1)}-c_{k-1}{ }^{(1)}\right)
$$

is implied by the convergence of the series

$$
\sum_{k=1}^{\infty}\left|c_{k}^{(i)}-c_{k-1}^{(i)}\right|, \quad(i=2,3, \cdots, n),
$$

and the existence of the limits

$$
\begin{gathered}
\lim _{k=\infty} c_{k}^{(1)} c_{k}{ }^{(2)} \cdots c_{k}{ }^{(n-1)}, \\
\lim _{k=\infty} c_{k}{ }^{(1)} \cdots c_{k}^{(i-1)} c_{k-1}{ }^{(i+1)} \cdots c_{k-1}{ }^{(n)},(i=2, \cdots, n-1) .
\end{gathered}
$$

In the special case when $n=2$ let $c_{k}{ }^{(1)}$ be the sum of the first $k-1$ terms of the converging infinite series $\alpha_{1}+\alpha_{2}+$ $\cdots$, so that $c_{k}^{(1)}-c_{k-1}{ }^{(1)}=\alpha_{k-1}$. Then we have the following theorem: If the series $\Sigma_{k} \alpha_{k}$ and $\Sigma_{k}\left|c_{k}{ }^{(2)}-c_{k-1}{ }^{(2)}\right|$ converge, so does also the series $\Sigma_{k} \alpha_{k} c_{k}{ }^{(2)}$. Owing to its wide and fundamental use in the theory of Dirichlet series, factorial series, and more general classes of series, this special case of our Theorem I must now be considered one of the classic tools of mathematical analysis.

The same identity (2) can be made to yield another general theorem of which the instance $n=2$ is also classic. It may be stated as follows:

TheOREm II. The convergence of the series

$$
\sum_{k=1}^{\infty} c_{k-1}{ }^{(2)} c_{k-1}{ }^{(3)} \cdots c_{k-1}{ }^{(n)}\left(c_{k}^{(1)}-c_{k-1}{ }^{(1)}\right)
$$

is implied by the convergence of the series

$$
\sum_{k=1}^{\infty}\left|c_{k}{ }^{(i)}-c_{k-1}{ }^{(i)}\right|, \quad(i=2,3, \cdots, n),
$$

the existence of the limit $\lim _{k=\infty} c_{k}{ }^{(1)} c_{k}^{(2)} \cdots c_{k}^{(n)}$, and the existence of a constant $B$ (independent of $k$ and $i$ ) such that for every $k$ we have

$$
\begin{array}{r}
\left|c_{k}^{(1)} c_{k}^{(2)} \cdots c_{k}^{(n-1)}\right|<B, \quad\left|c_{k}^{(1)} \cdots c_{k}^{(i-1)} c_{k-1}^{(i+1)} \cdots c_{k-1}^{(n)}\right| \\
<B \text { for } i=2,3, \cdots, n .
\end{array}
$$

On taking $c_{k}^{(i)}=c_{k}$ for $i=2,3, \cdots, n$, and writing $\nu$ for 
$n-1$, we have the following special case which deserves separate statement:

The convergence of the series

$$
\sum_{k=1}^{\infty}\left(c_{k-1}\right)^{\nu}\left(c_{k}{ }^{(1)}-c_{k-1}{ }^{(1)}\right)
$$

is implied by the convergence of the series $\Sigma_{k}\left|c_{k}-c_{k-1}\right|$ and either of the two following conditions:

(1) the existence of the limits

$$
\lim _{k=\infty} c_{k}^{(1)}\left(c_{k}\right)^{\nu-i}\left(c_{k-1}\right)^{i-1}, \quad(i=1,2, \cdots, \nu) ;
$$

(2) the existence of the limit $\lim _{k=\infty} c_{k}^{(1)}\left(c_{k-1}\right)^{\nu}$ and the existence of a constant $B$ (independent of $k$ and $i$ ) such that for every $k$ we have

$$
\left|c_{k}^{(1)}\left(c_{k}\right)^{\nu-i}\left(c_{k-1}\right)^{i-1}\right|<B, \quad(i=1,2, \cdots, \nu) .
$$

If the quantities $c_{k}{ }^{(i)}$ are not constants but are functions of a variable $x$ on a linear interval or in a domain of the complex plane, then it is clear that the series whose convergence is asserted in these theorems will converge uniformly if the conditions in the hypotheses are satisfied uniformly. This means, in Theorem I, that the limits explicitly mentioned and those implied by the convergent series in the hypothesis shall exist uniformly. In the case of Theorem II it means that the limits stated or implied in the hypothesis shall exist uniformly and that the inequalities shall be valid for a $B$ independent not only of $k$ and $i$ but also of $x$. The instance $n=2$ of each of these theorems is now also to be considered classic.

If in (1) we set the $a$ 's equal to unity and in the resulting identity sum as to $k$ from 1 to $m$, we have

$$
\begin{aligned}
\sum_{k=1}^{m}\left(c_{k}{ }^{(1)} c_{k}{ }^{(2)} \cdots c_{k}^{(n)}-1\right) & =\sum_{k=1}^{m}\left(c_{k}^{(1)}-1\right) \\
& +\sum_{i=2}^{n} \sum_{k=1}^{m} c_{k}^{(1)} c_{k}^{(2)} \cdots c_{k}^{(i-1)}\left(c_{k}{ }^{(i)}-1\right) .
\end{aligned}
$$

From this we obtain the following theorem:

Theorem III. Suppose that the series

$$
\sum_{k=1}^{\infty}\left|c_{k}{ }^{(i)}-1\right|, \quad(i=2,3, \cdots, n),
$$


converge; then if either of the series

$$
\sum_{k=1}^{\infty}\left(c_{k}^{(1)} c_{k}^{(2)} \cdots c_{k}^{(n)}-1\right) \text { and } \sum_{k=1}^{\infty}\left(c_{k}^{(1)}-1\right)
$$

converges, the other does also.

A similar theorem for uniform convergence may be formulated in an obvious manner.

If we examine the first two of the foregoing theorems in relation to identity (2), we see that the hypothesis in each case is framed so as to secure simply the existence of the limit $\lim _{k=\infty}$ $c_{k}{ }^{(1)} c_{k}{ }^{(2)} \cdots c_{k}{ }^{(n)}$ and the convergence of the series

$$
\begin{gathered}
\sum_{k=1}^{\infty} c_{k}{ }^{(1)} \cdots c_{k}{ }^{(n-1)}\left(c_{k}{ }^{(n)}-c_{k-1}{ }^{(n)}\right), \\
\sum_{k=1}^{\infty} c_{k}{ }^{(1)} \cdots c_{k}{ }^{(i-1)} c_{k-1}{ }^{(i+1)} \cdots c_{k-1}{ }^{(n)}\left(c_{k}{ }^{(i)}-c_{k-1}{ }^{(i)}\right), \\
(i=2,3, \cdots, n-1) ;
\end{gathered}
$$

moreover, the existence of this limit and the convergence of these series are sufficient to secure the convergence of the series whose convergence is asserted. Now it will be observed that the series (4) are separately of the same form as that whose convergence is asserted in the first two theorems. Hence these theorems themselves may be used for testing the convergence of series (4); and similarly for the auxiliary series employed in applying these theorems to series (4). Thus we have a sequence of theorems for testing convergence. To state them in general in terms of a parameter would call for complicated formulas; these will not be given, since it is probably just as expeditious to use the given theorems repeatedly. For the case $n=2$, however, the generalizations may be simply stated; these and others have been given in this Bulletin, volume 20 (1914), pages 225-233 and in Tôhoku Mathematical Journal, volume 11 (1917), pages 191-199.

I was led to a consideration of identity (1) in connection with the problem of integration of a Stieltjes integral by parts. It is of interest to treat that problem in connection with the foregoing, particularly since it may be used to illustrate the fact that a generalization of Abel's transformation of series is afforded by identity (1).

Let $\pi$ be a partition of the interval ( $a b)$ of norm $\delta$ due to the points $x_{0}=a, x_{1}, x_{2}, \cdots, x_{n-1}, x_{n}=b$ and let $\xi_{i}$ be a point of $\left(x_{i-1}, x_{i}\right)$. We have the identity 


$$
\begin{array}{r}
\sum_{i=1}^{n} f\left(\xi_{i}\right)\left\{v_{1}\left(x_{i}\right) v_{2}\left(x_{i}\right)-v_{1}\left(x_{i-1}\right) v_{2}\left(x_{i-1}\right)\right\} \equiv \sum_{i=1}^{n} f\left(\xi_{i}\right) v_{1}\left(x_{i}\right)\left[v_{2}\left(x_{i}\right)\right. \\
\left.-v_{2}\left(x_{i-1}\right)\right]+\sum_{i=1}^{n} f\left(\xi_{i}\right) v_{2}\left(x_{i-1}\right)\left[v_{1}\left(x_{i}\right)-v_{1}\left(x_{i-1}\right)\right]
\end{array}
$$

If we take $\xi_{i}=x_{i}$ and let $\delta$ approach zero, this relation yields the equation

$$
\begin{aligned}
\int_{a}^{b} f(x) d\left\{v_{1}(x) v_{2}(x)\right\}=\int_{a}^{b} f(x) v_{1}(x) & d v_{2}(x) \\
& +\int_{a}^{b} f(x) v_{2}(x) d v_{1}(x),
\end{aligned}
$$

provided that these Stieltjes integrals exist and that

(6) $\lim _{\delta=0} \sum_{i=1}^{n} v_{2}\left(x_{i-1}\right)\left[f\left(x_{i}\right)-f\left(x_{i-1}\right)\right]\left[v_{1}\left(x_{i}\right)-v_{1}\left(x_{i-1}\right)\right]=0$.

By taking $\xi_{i}=x_{i-1}$ and letting $\delta$ approach zero we have the same relation (5) provided that the integrals in it exist and that

$$
\lim _{\delta=0} \sum_{i=1}^{n} v_{1}\left(x_{i}\right)\left[f\left(x_{i}\right)-f\left(x_{i-1}\right)\right]\left[v_{2}\left(x_{i}\right)-v_{2}\left(x_{i-1}\right)\right]=0 .
$$

Now if $f(x)$ is continuous on $(a b)$ and $v_{1}(x)\left[v_{2}(x)\right]$ is of bounded variation on $(a b)$ while $v_{2}(x)\left[v_{1}(x)\right]$ is bounded, it is obvious that (6) $[(7)]$ is a valid relation. Thus we have sufficient conditions for the validity of (5) in case the integrals in (5) exist.

In particular, if $f(x)$ and either $v_{1}(x)$ or $v_{2}(x)$ are continuous on $(a b)$ and both $v_{1}(x)$ and $v_{2}(x)$ are of bounded variation on $(a b)$, then the integrals in (5) certainly exist and that relation is a valid one.

Let $f(x)$ be identically equal to 1 and write $g(x)$ for $v_{1}(x)$ and $v(x)$ for $v_{2}(x)$. Then on interchanging the members in (5) we have

(8) $\int_{a}^{b} g(x) d v(x)+\int_{a}^{b} v(x) d g(x)=g(b) v(b)-g(a) v(a)$,

provided these integrals exist. This special case of (5) is one of the frequently used formulas in the theory of Stieltjes integration; it is a generalization of the theorem for integration by parts in the theory of Cauchy-Riemann integration. 
The identity just preceding relation (5) is a generalization of that involved in the classic transformation of Abel; for, if we replace $v_{1}(x)$ by $g(x), v_{2}(x)$ by $v(x)$, and $f(x)$ by 1 , we have the Abel identity

$$
\begin{aligned}
\sum_{i=1}^{n} g\left(x_{i}\right)\left[v\left(x_{i}\right)-v\left(x_{i-1}\right)\right] & \\
& \equiv-\sum_{i=1}^{n} v\left(x_{i-1}\right)\left[g\left(x_{i}\right)-g\left(x_{i-1}\right)\right]+g(b) v(b)-g(a) v(a) .
\end{aligned}
$$

It is obvious that a repeated use of (5) reduces the integral of $f(x)$ as to a product $v_{1}(x) v_{2}(x) \cdots v_{n}(x)$ to a sum of $n$ integrals of functions as to $v_{1}(x), v_{2}(x), \cdots, v_{n}(x)$, respectively, under appropriate conditions like (6) and (7) and the hypothesis of the existence of these $n$ integrals. The question arises naturally as to whether some simple identity exists, analogous to that employed in deriving (5), which would yield the entire result at once. It was through this question that I was led to identity (1). Having it, it is natural to extend the classic theorems about convergence of series previously obtained through the particular case which yields Abel's transformation. The reader will have no difficulty in obtaining through identity (1) the transformation of a Stieltjes integral mentioned at the beginning of this paragraph.

UNIVERSITY OF ILLINOIS, August, 1919.

\section{NOTE ON A PHYSICAL INTERPRETATION OF STIELTJES INTEGRALS.}

BY PROFESSOR R. D. CARMICHAEL.

(Read before the American Mathematical Society September 4, 1919.)

Stifltues was led to his definition of integral by what he called the problem of moments (see $\$ 24$ of his memoir in Annales de la Faculté des Sciences de Toulouse, 1894). Consider on a straight line $O X$ a distribution of (positive) mass, the mass $m_{i}$ being concentrated at the distance $\xi_{i}$ from the origin 0 . The sum $\Sigma m_{i} \xi_{i}{ }^{k}$ he called the moment of order $k$ of the mass with respect to the origin. He aiso considered the more general distribution of mass on $O X$ which is such that 